\title{
CONJUGATE BUT NON INNER CONJUGATE SUBFACTORS
}

\author{
MARIE CHODA
}

(Communicated by Palle E. T. Jorgensen )

\begin{abstract}
It is shown that for each $r \in\left\{4 \cos ^{2}(\pi / n): n \geq 3\right\} \cup[4, \infty)$, there exist at least infinitely many subfactors of the hyperfinite $\mathrm{II}_{1}$ factor $R$ with index $r$, which are pairwise conjugate but non inner conjugate. In the case that $r$ is an integer, we have uncountably many such subfactors of $R$.
\end{abstract}

\section{INTRODUCTION}

Two von Neumann subalgebras $B$ and $C$ of a von Neumann algebra $A$ are said to be conjugate if there exists a ${ }^{*}$-automorphism $\theta$ of $A$ with $\theta(B)=C$. If we can take the $\theta$ as an inner automorphism of $A$, then $B$ and $C$ are said to be inner conjugate.

Kosaki and Yamagami showed in [12], among other things, that there are infinitely many inner conjugacy classes of subfactors in the hyperfinite $\mathrm{II}_{1}$ factor $R$ with the Jones index 2 ([10]). Otherwise, the subfactors of $R$ with index 2 are given as the fixed point algebras outer automorphisms on $R$ with the period $2([10])$ so that they are all conjugate by [4]. Bearing this fact in mind, we generalize their result in two directions.

One is that, for a given $r \in\left\{4 \cos ^{2}(\pi / n): n \geq 3\right\} \cup[4, \infty)$, there exists a countable infinity of non inner conjugate but conjugate subfactors of $R$ with index $r$. More precisely,

Theorem 1. Let $P$ be a subfactor of the hyperfinite $\mathrm{II}_{1}$ factor $R$ with $[R: P]<\infty$. Assume that either $[R: P] \leq 4$ (except one of type $A_{1}^{(1)}$ ) or $P \subset R$ is Jones' inclusion given in [10]. Then there exists a countable infinity of subfactors of $R$ which are pairwise non inner conjugate but conjugate to $P$.

A similar result holds for the subfactors of Wenzl ([21]), of Wassermann ([20]) and of Pimsner-Popa $([15,16])$. We construct such subfactors by an easy method (i.e. tensor product). To distinguish the inner conjugacy classes of these subfactors, we use basic (in the sense of [2]) *-endomorphisms of $R$ instead of bimodules as in $[12]$.

Another generalization is that for each integer $n$, there exist uncountably many non inner conjugate subfactors of $R$ which are pairwise conjugate. More precisely,

Theorem 2. Let $G$ be a finite group and $\alpha$ the outer action of $G$ on the hyperfinite $\mathrm{II}_{1}$ factor $R$. Then there exist uncountably many subfactors of $R$ which are pairwise non inner conjugate but conjugate to the fixed point algebra $R^{\alpha}$ of $G$ under $\alpha$.

Received by the editors April 4, 1994 and, in revised form, July 7, 1994.

1991 Mathematics Subject Classification. Primary 46L37; Secondary 46L40.

Key words and phrases. Hyperfinite $\mathrm{II}_{1}$ factor, index. 


\section{BASIC *-ENDOMORPHISMS}

Throughout this paper, $M$ is a $\mathrm{II}_{1}$ factor with separable predual and $\tau$ is the unique faithful normal trace of $M, \tau(1)=1$. We denote by $\operatorname{End}(M, \tau)$ (resp. $\operatorname{Aut}(M))$ the set of trace-preserving *-endomorphisms (resp. automorphisms) of $M$. If $\sigma \in \operatorname{End}(M, \tau)$, then $\sigma$ is unit-preserving, faithful and $\sigma$-weakly continuous. Hence $\sigma(M)$ is a subfactor of $M$. Assume that the index $[M: \sigma(M)]<\infty$. By iterating the Jones basic construction ([10]) from the inclusion $\sigma(M) \subset M$, the tower of $\mathrm{II}_{1}$ factors $\left\{M_{n}\right\}_{n \geq 1}$ is obtained:

$$
\sigma(M) \subset M \subset M_{1}=\left\langle M, e_{1}\right\rangle \subset \cdots \subset M_{n}=\left\langle M_{n-1}, e_{n}\right\rangle \cdots,
$$

where $e_{n}$ is the Jones projection for the inclusion $M_{n-2} \subset M_{n-1}$. The trace $\tau$ is extended to the unique trace $\tau$ of $M_{n}(n \geq 1)$. Let $M_{\infty}$ be the $\mathrm{II}_{1}$ factor obtained from the GNS construction of $\bigcup_{n} M_{n}$ by $\tau$.

A $\sigma \in \operatorname{End}(M, \tau)$ is called basic ([2]) if $[M: \sigma(M)]<\infty$ and $\sigma$ satisfies one of the following equivalent conditions:

(1) $\sigma$ is extended to a ${ }^{*}$-isomorphism from $M_{1}$ onto $M$.

(2) There exists a projection $e \in M$ such that

$$
\sigma^{2}(M)=\{e\}^{\prime} \cap \sigma(M), \quad E_{\sigma(M)}(e)=[M: \sigma(M)]^{-1},
$$

where $E_{B}$ is the $\tau$-preserving conditional expectation of $M$ onto the von Neumann subalgebra $B$ of $M$. Such a projection $e \in M$ is called a basic projection for $\sigma$.

Two *-endomorphisms $\rho$ and $\sigma$ of $M$ are said to be unitarily equivalent if there exists a unitary $u \in M$ with $u \rho(a) u^{*}=\sigma(a)(a \in M)$. The unitary equivalence class of $\rho$ is denoted by $[\rho]$. Then

(i) if $[\rho]=[\sigma]$, then $\left[\rho^{n}\right]=\left[\rho^{n-1} \sigma\right]$, for all $n$;

(ii) if $[\rho]=[\sigma \cdot \theta]$ for some $\theta \in \operatorname{Aut}(M)$, then $\rho(A)^{\prime} \cap A$ is inner conjugate to $\sigma(A)^{\prime} \cap A$.

Example 1. Let $A_{0}$ be the $m \times m$ matrices and $M=\bigotimes_{i=1}^{\infty}\left(A_{i}, \tau_{i}\right)$, where $A_{i}=$ $A_{0}$, and $\tau_{i}$ is the normalized unique trace of $A_{i}(i \geq 1)$. Let $\eta_{i}$ be the canonical embedding of $A_{0}$ onto $A_{i}$ (under the identification with the subfactor of $M$ ) with $\eta_{i}(x)=1 \otimes \cdots \otimes 1 \otimes x \otimes 1 \otimes \cdots$. Then the ${ }^{*}$-endomorphism $\sigma$ of $M$ defined by $\sigma\left(\eta_{i}(x)\right)=\eta_{i+1}(x)\left(x \in A_{0}\right)$ is basic.

Example 2 (Jones' inclusion). Let $r \in\left\{4 \cos ^{2}(\pi / n): n \geq 3\right\} \cup[4, \infty)$ and put $\lambda=r^{-1}$. Let $P$ be the factor generated by the sequence of projections $\left\{e_{i} ; i \geq 1\right\}$ with

$$
e_{i} e_{j} e_{i}= \begin{cases}\lambda e_{i} & (|i-j|=1) \\ e_{i} e_{j} & (|i-j| \neq 1)\end{cases}
$$

and $Q=\left\{e_{i}: i \geq 2\right\}^{\prime \prime}$. The inclusion $Q \subset P$ of hyperfinite $\mathrm{II}_{1}$ factors is called Jones' inclusion and satisfies that $[P: Q]=r, Q$ is irreducible (i.e. $Q^{\prime} \cap P=\mathbf{C} 1$ ) if $r \leq 4([10])$ and $\operatorname{dim}\left(Q^{\prime} \cap P\right)=2$ if $r>4$ ([15]). A basic *-endomorphism $\sigma$ of $P$ with $\sigma(P)=Q$ is defined by $\sigma\left(e_{i}\right)=e_{i+1}$.

Example 3 (Wenzl's inclusion). The method in Example 2 is generalized by Wenzl ([21]), considering certain unitary representations $\pi$ of the Braid group on infinitely many generators $g_{0}, g_{1}, \cdots$ that admit positive Markov traces. The irreducible inclusion $N \subset M$ is given as $N=\left\{\pi\left(g_{j}\right) ; j \geq 2\right\}^{\prime \prime} \subset M=\left\{\pi\left(g_{j}\right) ; j \geq 1\right\}^{\prime \prime}$. A basic *-endomorphism $\sigma$ of $M$ with $\sigma(M)=N$ is defined by $\sigma\left(\pi\left(g_{i}\right)\right)=\pi\left(g_{i+1}\right)(i \geq 1)$. 
Example 4 (Ocneanu's inclusion). The above examples are generalized using bipartite graphs by Ocneanu $([13,14])$. Let $\mathcal{G}$ be a finite bipartite graph with a biunitary connection $W$ with respect to the distinguished point * among the even vertices of $\mathcal{G}$. Considering the algebra $\operatorname{String}^{(n)} \mathcal{G}$ of $n$-strings on $\mathcal{G}$, we give a trace $\tau$ and an embedding $i_{n}^{n+k}$ of $\operatorname{String}^{(n)} \mathcal{G}$ into $\operatorname{String}^{(n+k)} \mathcal{G}$, which is compatible with $\tau$. The trace on String $^{(n)} \mathcal{G}$ which is compatible with the embedding is unique. The hyperfinite $\mathrm{II}_{1}$ factor $M(\mathcal{G})$ is obtained by the GNS construction of $\left(\bigcup_{n}\right.$ String $\left.^{(n)} \mathcal{G}\right)$ with respect to $\tau$. Another embedding $\varphi$ of $\operatorname{Strings}^{(n)}(\mathcal{G})$ into $\operatorname{Strings}^{(n+1)}(\mathcal{G})$ different with $i_{n}^{n+1}$ is induced by the unitary matrix in the biunitary axiom of $W$. The $\varphi$ is a ${ }^{*}$-isomorphism compatible with the inclusion $i_{n}^{n+k}$ and preserves $\tau$ so that it is extended to $M(\mathcal{G})$. Ocneanu's subfactor $N(\mathcal{G})$ is nothing else but $\varphi(M(\mathcal{G})$ ). The $\varphi$ is a basic *-endomorphism of $M(\mathcal{G})$.

Example 5 (Pimsner-Popa's inclusion). Let $\sigma$ be the basic *-endomorphism of $M$ in Example 1 for $m=2$ and $\eta_{i}$ be the same as in Example 1. Let $\theta$ be an outer automorphism of $M$. Put

$$
\rho(x)=\eta_{1}\left(e_{11}\right) \sigma(x)+\eta_{1}\left(e_{22}\right) \sigma(\theta(x)) \quad(x \in M),
$$

where $\left\{e_{i j}\right\}_{i, j=1,2}$ is the canonical matrix unit of $M_{2}(\mathbf{C})$. Then $\rho \in \operatorname{End}(M, \tau)$. The subfactor $N=\rho(M)$ is Pimsner-Popa's subfactor $([15,17])$ associated with $\theta$. Since $[\sigma]=\left[\theta^{-1} \cdot \sigma \cdot \theta\right], \rho$ is a basic *-endomorphism of $M$ with $\rho(M)=N$.

Example 6 (Wasserman's inclusion). Let $M$ and $\sigma$ be the same as in Example 5 . Let $G$ be a subgroup of $S U(2)$. Then $G$ acts on $M$ by the infinite tensor product of its action by conjugation on $M_{2}(\mathbf{C})$. Let $A=\left(M_{2}(\mathbf{C}) \otimes M\right)^{G} \supset B=(1 \otimes M)^{G}$. Then $N$ is a subfactor of $M$ with index 4 and the principal graph is obtained as one of the extended Coxeter graph corresponding $G$ 's $([5,20])$. Since the action of $G$ commutes with $\sigma, \sigma$ becomes a basic *-endomorphism of $M$ with $\sigma(M)=N$.

Proposition 1. Let $N \subset M$ be an inclusion of hyperfinite $\mathrm{II}_{1}$ factors with $[M$ : $N] \leq 4$. Then there exists a basic *-endomorphism $\sigma$ of $M$ with $\sigma(M)=N$.

Proof. The complete classification of subfactors with index $\leq 4$ of the hyperfinite $\mathrm{II}_{1}$ factor is established in [18]. The principal graph of $N \subset M$ is one of the Coxeter graphs $A_{n}(n \geq 3), D_{2 n}(n \geq 2), E_{6}, E_{8}$ and the extended Coxeter graphs $A_{n}^{(1)}(2 \leq n \leq \infty), D_{n}^{(1)}(n \geq 4), A_{\infty}, D_{\infty}, E_{6}^{(1)}, E_{7}^{(1)}, E_{8}^{(1)}([1,5,6,7,8,10,11,13$, $14,16,17,19])$.

The subfactor of type $A_{n}, 3 \leq n<\infty$, is unique $([10,11,13,14,16])$ and it is a Jones' subfactor so that a basic *-endomorphism is given in Example 2. The subfactors of types $D_{2 n}, E_{6}, E_{8}, D_{n}^{(1)}$ are constructed by the method of string algebras via flat connections due to Ocneanu $([7,8,11,13,14])$ and they respectively have basic *-endomorphisms in Example 4. Each inclusion of type $A_{2 n-1}^{(1)}(1 \leq n<$ $\infty)$ is given as Pimpner-Popa's inclusion $M \supset N$ associated with the $\theta=s_{n}^{\gamma}$ by Connes ([4]) for some $\gamma$ with $\gamma^{n}=1([14,17])$. For $A_{\infty}^{(1)}$, the inclusion is unique and the unique (up to the outer conjugacy) aperiodic automorphism ([3]) corresponds to $\theta$ ([17]). Hence a basic *-endomorphism of $M$ of type $A_{2 n-1}^{(1)}(1 \leq n \leq \infty)$ with $N$ as its range is given as $\rho$ in Example 5. The subfactor with one of $E_{6}^{(1)}, E_{7}^{(1)}, E_{8}^{(1)}$ 
as the principal graph is unique ([11]), so it is given by Wasserman's method ([5]). Hence it has a basic *-endomorphism in Example 6.

Thus each subfactor is given as the range of a basic *-endomorphism.

\section{Non inner CONJUGATE SUbFaCtors}

In this section, we distinguish subfactors of the hyperfinite $\mathrm{II}_{1}$ factor which are given through the method of the tensor product by using unitary equivalence relations of basic *-endomorphisms.

Lemma 2. Let $\rho, \sigma \in \operatorname{End}(M, \tau)$. Then $\rho(M)$ is inner conjugate to $\sigma(M)$ if and only if there exists a $\theta \in \operatorname{Aut}(M)$ with

$$
[\sigma \cdot \theta]=[\rho] .
$$

Proof. Assume $u \rho(M) u^{*}=\sigma(M)$ for some unitary $u \in M$. Then $\sigma^{-1}\left(u \rho(M) u^{*}\right)=$ $M$. Putting $\theta=\sigma^{-1} \cdot \operatorname{Ad}(u) \cdot \rho$, we have $\theta \in \operatorname{Aut}(M)$ and $[\sigma \cdot \theta]=[\rho]$.

Lemma 3. Let $R$ be the hyperfinite $\mathrm{II}_{1}$ factor with the canonical trace $\tau$ and a basic $\sigma$ have $[R: \sigma(R)]=r<\infty$. Put

$$
M=\bigotimes_{m \in \mathbf{Z}}\left(R_{m}, \tau_{m}\right), \quad M(i)=\bigotimes_{j<i}\left(R_{j}, \tau_{j}\right) \otimes\left(\sigma_{i}(R), \tau_{i}\right) \otimes \bigotimes_{i<k}\left(R_{k}, \tau_{k}\right),
$$

where $R_{m}=R, \tau_{m}=\tau, \sigma_{m}=\sigma(m \in \mathbf{Z})$. Assume that $\sigma$ satisfies the condition $(*)$ :

$\left(^{*}\right) \quad \exists n \in \mathbf{N}: \sigma^{n}(R)^{\prime} \cap R$ is not isomorphic to $\left(\sigma^{n-1}(R)^{\prime} \cap R\right) \otimes\left(\sigma(R)^{\prime} \cap R\right)$.

Then the subfactors $\{M(i): i \in \mathbf{Z}\}$ of $M$ are pairwise conjugate but non inner conjugate.

Proof. Let

$$
\rho_{i}=\bigotimes_{j<i} \operatorname{id}_{j} \otimes \sigma_{i} \otimes \bigotimes_{i<k} \operatorname{id}_{k}
$$

Then $\rho_{i}$ is a basic *-endomorphism of $M$ with $\rho_{i}(M)=M(i)$ and $\left[M: \rho_{i}(M)\right]=$ $[R: P]$. It is clear that $\rho_{i}(M)$ is conjugate to $\rho_{k}(M)$ by the automorphism of $M$ which flips the $i$-th factor and the $k$-th factor of $M$. Assume that $\rho_{i}(M)$ is inner conjugate to $\rho_{k}(M)$ for $i \neq k$. Then there exists a $\theta \in \operatorname{Aut}(M)$ such that $\left[\rho_{i} \cdot \theta\right]=\left[\rho_{k}\right]$ by Lemma 2. Hence $\rho_{k}^{n}(M)^{\prime} \cap M$ is isomorphic to $\rho_{k}^{n-1}\left(\rho_{i}(M)\right)^{\prime} \cap M$ by (i) and Lemma 2. On the other hand, $\rho_{k}^{n}(M)^{\prime} \cap M$ is isomorphic to $\sigma^{n}(R)^{\prime} \cap R$, but $\rho_{k}^{n-1}\left(\rho_{i}(M)\right)^{\prime} \cap M$ is isomorphic to $\left(\sigma^{n-1}(R)^{\prime} \cap R\right) \otimes\left(\sigma(R)^{\prime} \cap R\right)$. This contradicts the assumption.

Proof of Theorem 1. By the assumption, either $[R: P] \leq 4$ or $P \subset R$ is Jones' inclusion. Hence by Proposition 1 and Example 2, we have a basic $\sigma \in \operatorname{End}(R, \tau)$ with $\sigma(R)=P$.

For such a pair $\{R, \sigma\}$, let $M$ and $M(i)$ be the same as in Lemma 3 . It is sufficient to prove that the family $\{M(i)\}_{i \in \mathbf{Z}}$ is pairwise non inner conjugate but $M(i) \subset M$ is isomorphic to $P \subset R$, that is, there is a *-isomorphism from $M$ onto $R$ which transforms $M(i)$ onto $P$. 
First we show that $\sigma$ satisfies the condition $(*)$ in Lemma 3. Since $\sigma$ is basic, the $n$-th basic extension for $P \subset R$ is given as $\sigma^{-n}(R)$. If the inclusion $P \subset R$ is irreducible, then (*) is satisfied by $n=2$ because $\sigma^{2}(R)^{\prime} \cap R$ contains a basic projection for $\sigma$. Hence $\left(^{*}\right)$ is satisfied if $P \subset R$ is one of the cases: $[R: P]<4$, or the type of $A_{\infty}$ (Jones' inclusion of index 4$), D_{n}^{(1)}, D_{\infty}, E_{n}^{(1)}(n=6,7,8)$. If the principal graph of $P \subset R$ is $A_{n}^{(1)}(2 \leq n \leq \infty)$, then $\sigma(R)^{\prime} \cap R$ is a 2-dimensional algebra, so that $\left(\sigma(R)^{\prime} \cap R\right) \otimes\left(\sigma(R)^{\prime} \cap R\right)$ is abelian but $\sigma^{2}(R)^{\prime} \cap R$ is not abelian; thus $(*)$ is satisfied by $n=2$. The principal graph of Jones' inclusion with index $>4$ is also $A_{\infty}^{(1)}([5])$. Thus $\left(^{*}\right)$ is satisfied by $n=2$ for all inclusions. Hence $M(i)$ is not inner conjugate to $M(k)$ for $i \neq k$ by Lemma 3 .

Next, we show that $M(i) \subset M$ is isomorphic to $P \subset R$. Remark that the inclusion $M(i) \subset M$ has the tower of the relative commutants in the basic extensions isomorphic to that of $P \subset R$. Popa shows that if $N \subset M$ is an extremal and strongly amenable inclusion of hyperfinite $\mathrm{II}_{1}$ factors, then $N \subset M$ is opposite isomorphic to $M_{1}^{\prime} \cap M_{\infty} \subset M^{\prime} \cap M_{\infty}([18,5.1 .2$ Corollary $])$ and that $N \subset M$ is extremal and strongly amenable if $[M: N] \leq 4$. Hence $M(i) \subset M$ is isomorphic to $P \subset R$ if $[R: P] \leq 4$. If $P \subset R$ is Jones' inclusion with $[R: P]>4$, then

$$
M(i)^{\prime} \cap M=\mathbf{C} p+\mathbf{C}(1-p)
$$

for a projection $p \in M$ with $\tau(p) \tau(1-p)=[M: M(i)]^{-1}$ and $\operatorname{dim}\left(M(i)^{\prime} \cap M\right)=2$. Hence, $M(i) \subset M$ is isomorphic to $P \subset R$ by the characterization of locally trivial subfactors ([18, 5.2.3 Corollary $])$. Therefore for each given inclusion, $M(i) \subset M$ is isomorphic to $P \subset R$.

Remark. The proof of Theorem 1 also holds for Wenzl's inclusion and the other inclusion which has the paragroup as the conjugacy invariant.

The most typical subfactor of a finite factor $M$ with finite index are given as the fixed point algebra $M^{\alpha}$ under the outer action $\alpha$ of a finite group $G$. Next we discuss such subfactors.

Proposition 4. Let $G$ be a finite group and $\alpha$ an outer action of $G$ on $M$. If $G$ is nonabelian, then there are no basic *-endomorphisms of $M$ which have the subfactor $M^{\alpha}$ as the range.

Proof. Assume there exists a basic *-endomorphism $\sigma$ of $M$ with $\sigma(M)=M^{\alpha}$. Let $M_{\infty}$ be the enveloping factor obtained from the tower of Jones' basic extension algebra for the inclusion $M^{\alpha} \subset M$. Then we have a $\beta \in \operatorname{Aut}\left(M_{\infty}\right)$ with $\beta\left(M_{i}\right)=$ $M_{i-1}$ for all $i$, which is the extension of $\sigma$. Hence the principal graph and the dual principal graph for $M^{\alpha} \subset M$ are isomorphic. This contradicts that $G$ is nonabelian.

By Proposition 4, the proof of Theorem 1 does not work for the inclusion $M^{\alpha} \subset$ $M$ to the outer action of a nonabelian finite group $G$. In the case of fixed point algebras of finite groups, we use the outerness of actions to distinguish subfactors.

Proof of Theorem 2. Let $I$ and $J$ be subsets of $\mathbf{Z}$ with $I \neq J$. Let

$$
\begin{gathered}
M=\bigotimes_{m \in Z}\left(R_{m}, \tau_{m}\right), \\
\beta(g)=\bigotimes_{i \in I} \alpha_{i}(g) \otimes \bigotimes_{i \in \mathbf{Z} \backslash I} \operatorname{id}_{i}, \quad \gamma(g)=\bigotimes_{i \in J} \alpha_{i}(g) \otimes \bigotimes_{i \in \mathbf{Z} \backslash J} \mathrm{id}_{i} .
\end{gathered}
$$


Here $R_{m}=R, \tau_{m}=\tau$ (the canonical trace) of $R$ and $\alpha_{m}=\alpha(m \in \mathbf{Z})$.

We denote the canonical trace on $M$ by the same notation $\tau$. Then $\beta$ and $\gamma$ are outer actions of $G$ on $M$. Since the outer action of $G$ on the hyperfinite $\mathrm{II}_{1}$ factor is unique up to conjugacy ([9]), $M^{\beta} \subset M$ is conjugate to $R^{\alpha} \subset R$ for all $I \subset \mathbf{Z}$. Assume that $u M^{\beta} u^{*}=M^{\gamma}$ for some $u \in M$. Let $v$ (resp. $w$ ) be the unitary representation of $G$ on $L^{2}(M, \tau)$ such that $\beta(g)=\operatorname{Ad} v(g)(\operatorname{resp} . \gamma(g)=\operatorname{Ad} w(g))$. Then $\left(u v(G) u^{*}\right)^{\prime} \cap$ $M=u\left(v(G)^{\prime} \cap M\right) u^{*}=w(G)^{\prime} \cap M$. Put $L=M^{\prime} \cap B\left(L^{2}(M, \tau)\right)$. Then two crossed products coincide: $L \triangleleft_{u \beta} G=L \triangleleft_{\gamma} G$, where $(u \beta)(g)=\operatorname{Ad}\left(u v(g) u^{*}\right)$ and $\gamma(g)=$ Ad $w(g)$ on $L$. Since the actions $u \beta$ and $\gamma$ of $G$ on $L$ are outer, there exists a conditional expectation $E$ of $L \triangleleft_{u \beta} G$ onto $L$ with $E(v(g))=E\left(u w(g) u^{*}\right)=0$ if $g \neq$ 1. Let $u v(g) u^{*}=\sum_{h \in G} w(h) x(h)(x(h) \in L)$ be the Fourier expansion of $u v(g) u^{*}$. Then $x(1)=0$, where 1 is the identity of $G$. Since $I \neq J,(u \beta)\left(g^{-1}\right) \gamma(h)$ is an outer automorphism of $L$ if $g \neq 1 \neq h$. Hence we have $1=\sum_{h \neq 1} u v\left(g^{-1}\right) u^{*} w(h) x(h)=$ $\sum_{h \neq 1} E\left(u v\left(g^{-1}\right) u^{*} w(h) x(h)\right)=0$. This is a contradiction.

\section{ADDENDUM}

A second referee writes that since $\operatorname{End}\left({ }_{M(i)} L^{2}(M)_{M(j)}\right)$ is not isomorphic to $\operatorname{End}\left({ }_{M(i)} L^{2}(M)_{M(i)}\right)$, without any assumptions on values of indices Theorem 1 is proved by Kosaki-Yamagami's bimodule method. Theorem 1 is shown so. However, in the case that $\sigma(R) \subset R$ is of type $A_{1}^{(1)}, M(i)$ is inner conjugate to $M(j)$.

\section{REFERENCES}

1. J. Bion-Nadal, An example of a subfactor of the hyperfinite $\mathrm{II}_{1}$ factor whose principal graph invariant is the Coxeter graph $E_{6}$, Current Topics in Operator Algebras, World Scientific, Singapore, 1991, pp. 104-113. MR 94a:46085

2. M. Choda, Entropy for canonical shifts, Trans. Amer. Math. Soc. 334 (1992), 827-849. MR 93k:46054

3. A. Connes, Outer conjugacy classes of automorphisms of factors, Ann. Sci. École Norm. Sup. 8 (1975), 383-419. MR 56:9277

4. $\ldots$, Periodic automorphisms of the hyperfinite factor of type $\mathrm{II}_{1}$, Acta Sci. Math. (Szeged) 39 (1977), 39-66. MR 56:6411

5. F. Goodman, P. de la Harpe, and V. F. R. Jones, Coxeter graphs and towers of algebras, Springer, New York, 1989. MR 91c:46082

6. M. Izumi, Application of fusion rules to classification of subfactors, Publ. Res. Inst. Math. Sci. 27 (1991), 953-994. MR 93b:46121

7. _ On flatness of the Coxeter graph $E_{8}$, Pacific J. Math. (to appear). CMP 95:07

8. M. Izumi and Y. Kawahigashi, Classification of subfactors with the principal graph $D_{n}^{(1)}$, J. Funct. Anal. 112 (1993), 257-286. MR 94k:46127

9. V. F. R. Jones, Actions of finite groups on the hyperfinite type $\mathrm{II}_{1}$ factor, Mem. Amer. Math. Soc., vol. 28, no. 237, Amer. Math. Soc., Providence, RI, 1980. MR 81m:46094

10. _ Index for subfactors, Invent. Math. 72 (1983), 1-15. MR 84d:46097

11. Y. Kawahigashi, On flatness of Ocneanu's connections on the Dynkin diagrams and classification of subfactors, J. Funct. Anal. 127 (1995), 63-107. CMP 95:05

12. H. Kosaki and S. Yamagami, Irreducible bimodules associated with crossed product algebras, Internat. J. Math. 5 (1992), 661-676. MR 94f:46087

13. A. Ocneanu, Quantized groups, string algebras and Galois theory for algebras, Operator Algebras and Applications, London Math. Soc. Lecture Note Ser., vol. 136, Cambridge Univ. Press, Cambridge, 1988, pp. 119-172. MR 91k:46068

14. __ Quantum symmetry, differential geometry of finite graphs and classification of subfactors, University of Tokyo Seminary Notes 45 (notes recorded by Y. Kawahigashi), 1991.

15. M. Pimsner and S. Popa, Entropy and index for subfactors, Ann. Sci. École Norm. Sup. 19 (1986), 57-106. MR 87m:46120 
16. S. Popa, Classification of subfactors: the reduction to commuting squares, Invent. Math. 101 (1990), 19-43. MR 91h:46109

17. Sur la classification des sous-facteurs d'indice fini du facteur hyperfini, C. R. Acad. Sci. Paris 311 (1990), 95-100. MR 91m:46094

18. Classification of amenable subfactors of type II, preprint. MR 95f:46105

19. V. S. Sunder and A. K. Vijayarajan, On the non-occurrence of the Coxeter graphs $\beta_{2 n+1}, E_{7}$, $D_{2 n+1}$ as principal graphs of an inclusion of $\mathrm{II}_{1}$ factor, Pacific J. Math. 161 (1993), 185-200. MR 94g:46067

20. A. Wassermann, Coactions and Yang-Baxter equations for ergodic actions and subfactors, Operator Algebras and Applications, London Math. Soc. Lecture Notes Ser., vol. 136, Cambridge Univ. Press, Cambridge, 1988, pp. 202-236. MR 92d:46167

21. H. Wenzl, Hecke algebras of type $A_{n}$ and subfactors, Invent. Math. 92 (1988), 349-383. MR 90b: 46118

Department of Mathematics, Osaka Kyoiku University, Asahigaoka, Kashiwara 582, JAPAN

E-mail address: foo527@sinet.ad.jp 